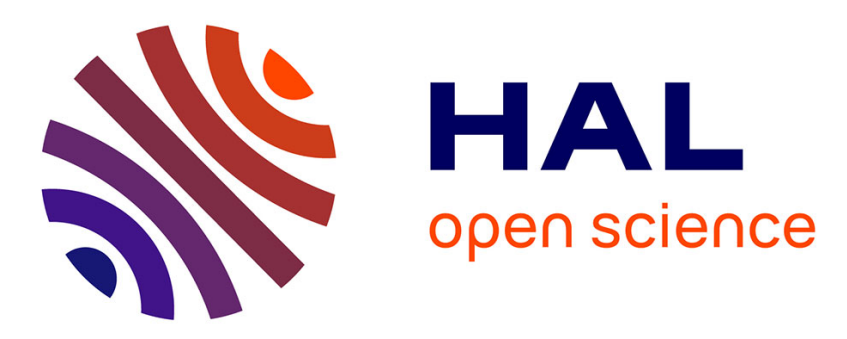

\title{
Comment réduire les dysphonies parkinsoniennes par thyroplastie
}

\author{
B. Roubeau, M. Bruel, O. de Crouy Chanel, Sophie Périé
}

\section{To cite this version:}

B. Roubeau, M. Bruel, O. de Crouy Chanel, Sophie Périé. Comment réduire les dysphonies parkinsoniennes par thyroplastie. Annales françaises d'Oto-rhino-laryngologie et de Pathologie Cervico-faciale, 2016, 133 (6), pp.398 - 400. 10.1016/j.aforl.2016.02.009 . hal-01484008

\section{HAL Id: hal-01484008 \\ https://hal.sorbonne-universite.fr/hal-01484008}

Submitted on 6 Mar 2017

HAL is a multi-disciplinary open access archive for the deposit and dissemination of scientific research documents, whether they are published or not. The documents may come from teaching and research institutions in France or abroad, or from public or private research centers.
L'archive ouverte pluridisciplinaire HAL, est destinée au dépôt et à la diffusion de documents scientifiques de niveau recherche, publiés ou non, émanant des établissements d'enseignement et de recherche français ou étrangers, des laboratoires publics ou privés. 
Comment réduire les dysphonies parkinsoniennes par thyroplastie

Bernard ROUBEAU, Magali BRUEL, Olivier de Crouy Chanel, Sophie PERIE*

Service d'ORL et de Chirurgie Cervico-faciale, Hôpital Tenon, Assistance Publique Hôpitaux de Paris, Université Pierre et Marie Curie, PARIS VI, 4 rue de la Chine, 75020 Paris, France

\section{* Auteur correspondant.}

Adresse e-mail : sophie.perie@tnn.aphp.fr (S. Périé)

Phone : $33156016417 \quad$ Fax : 33156017010 


\title{
RESUME
}

L'hypophonie joue un rôle important sur la qualité de la communication orale des patients parkinsoniens en majorant les effets de la dysarthrie associée. Lorsque cette hypophonie est liée à un défaut d'adduction des cordes vocales, constituant ainsi une «incompétence laryngée», une médialisation d'une corde vocale peut être proposée en cas d'échec de la prise en charge rééducative.

Des techniques d'analyses acoustique et aérodynamique de la voix et de la parole, d'évaluation perceptive et d'estimation du handicap vocal, associées à une fibroscopie laryngée ont été réalisées pour discuter de l'indication de médialisation laryngée dans ces incompétences laryngées.

Une médialisation d'une corde vocale par thyroplastie (implant de Montgomery), sous anesthésie locale et neuroanalgésie, a été réalisée chez deux patients parkinsoniens avec une dysphonie réfractaire à la rééducation.

Après l'intervention, une réévaluation des différents paramètres a montré un enrichissement du timbre via l'accroissement du nombre d'harmoniques et une amélioration des paramètres aérodynamiques.

La médialisation d'une corde vocale, en réduisant l'hypophonie par une meilleure adduction glottique, a amélioré efficacement la voix et le confort de communication orale de ces deux patients. La technique de médialisation par thyroplastie, sous anesthésie locale, permet un contrôle peropératoire de la voix mais aussi le retrait potentiel de l'implant.

\section{MOTS CLES}

Maladie de Parkinson, dysarthrie, hypophonie, médialisation laryngée, thyroplastie

\begin{abstract}
Parkinsonian hypophonia has an important impact on the loss of the quality of the speech with the evident consequences on the social patient's abilities. When this hypophonia is in relation to a persistent default of vocal folds adduction as a real "laryngeal insufficiency", even after an intensive voice and speech therapy, a medialization seems to be an interesting treatment. A medialization thyroplasty of one vocal fold by Montgomery implant with local anesthesia and neuroanalgesia was proposed to a woman of 62 and a man of 78 years old. Indications were based on acoustical and aerodynamic analyses of voice and speech, and on laryngeal
\end{abstract}


fiberoscopy. Perceptive scale was used and handicap evaluation was performed. The enriching of vocal quality by increasing the number of harmonics and by improving aerodynamic parameters were observed in both patients postoperatively.

Medialization of one vocal fold improves the glottal adduction and decreases the hypophonia which has a positive effect on the speech communication. Advantages of thyroplasty are local anesthesia, peroperative control of result and potential implant extraction.

\section{KEY WORDS}

Parkinson disease, dysarthria, hypophonia, laryngeal medicalization, thyroplasty 


\section{Introduction}

Le tableau clinique de la maladie de Parkinson comporte le plus souvent une dysarthriedysphonie abondamment décrite dans la littérature $(1,2)$ caractérisée notamment par une hypophonie et une hypokinésie articulatoire. Les déficiences phonatoires, articulatoires et prosodiques qui en découlent ont un retentissement systématique sur le niveau de communication orale des patients et la qualité de leurs relations sociales. Les traitements chimiques ont peu d'effet sur ces troubles de la voix et de la parole et seule la prise en charge rééducative permet de limiter les effets délétères de la maladie sur la parole des patients. Les prises en charges sont basées principalement sur un contrôle de l'intensité vocale ou du débit de parole et dans une moindre mesure sur le contrôle articulatoire (3). Un renforcement efficace de l'adduction laryngée par un entraînement volontaire soutenu, tel qu'il est pratiqué dans la méthode LSVT, améliore l'intensité vocale et s'accompagne automatiquement d'une amplification des mouvements articulatoires, ce qui réduit l'hypophonie et la dysarthrie $(4,5)$. Dans certains cas, le renforcement de l'adduction laryngée ne peut être obtenu de façon suffisante et se trouve responsable de l'échec de la prise en charge. Le larynx demeure incompétent et incapable d'accroître l'intensité sonore. Certains auteurs sont partis du principe que la réduction de l'hypophonie seule aurait déjà un impact positif sur l'efficacité de la parole des patients. Dans ce but, une médialisation laryngée en vue d'améliorer la fermeture glottique et d'optimiser la vibration laryngée a été proposée avec succès (6). Les techniques de médialisation décrites dans la littérature auprès de patients parkinsoniens font appel à une injection intracordale soit de collagène, soit de silicone $(7,8,9,10)$.

Les injections de collagène ou de silicone, du fait de l'irréversibilité de l'acte chirurgical peuvent, comporter un risque dans le cadre d'une maladie évolutive où un défaut de mobilité bilatérale peut survenir. D'autre part, pour certaines de ces techniques d'injections, l'implication d'une anesthésie générale, pouvant être un facteur limitant chez ces patients, nous a conduits à proposer une médialisation par thyroplastie (11). Le but était d'améliorer l'adduction laryngée chez deux patients atteints de maladie de parkinson en échec de thérapie rééducative de type LSVT.

\section{Technique}


La technique présentée comporte deux volets : d'une part, l'évaluation de la voix des patients, et d'autre part la médialisation laryngée par thyroplastie.

Une étude détaillée de la dysphonie était effectuée dans le but de poser l'indication de médialisation et d'évaluer les effets de cette technique opératoire.

L'analyse de la phonation comportait des évaluations instrumentales (tableaux I, II) et non instrumentales. L'étude acoustique de sons tenus consistait en une analyse spectrale de type sonagraphie avec comptabilisation des harmoniques dans une fenêtre comprise entre 0 à 6000 Hz. Pour l'analyse aérodynamique, la fuite glottique (11), ou débit trans-glottique, était mesurée et exprimée en $\mathrm{cc} / \mathrm{sec} / \mathrm{dB}$ lors de la tenue d'une voyelle, grâce à un équipement de type EVA 2 muni de capteurs de débit. Le temps maximum de phonation était mesuré lors de la production d'un /a/ tenu. Le volume d'air expiré en dm3 calculé à partir de l'intégration du débit glottique dans le temps était calculé lors de la production d'une phrase. Un test sous masquage était réalisé par une épreuve de lecture et de parole spontanée sous masquage auditif par un bruit blanc de $85 \mathrm{~dB}$. Le test était considéré négatif lorsqu'il n'existait pas d'accroissement efficace de l'intensité vocale par effet Lombard. Dans ce cas, l'indication de médialisation d'une corde vocale par thyroplastie était discutée et un examen laryngé fibroscopique était réalisé.

Les évaluations non instrumentales de la voix $(12,13)$ et du confort de la communication orale comprenaient le GBRAS (Grade global de la dysphonie, Bruit de souffle, Raucité, « Asthénie », Serrage) (grade de sévérité de 0 à 3), et le VHI 10 (14), questionnaire évaluant le handicap vocal par 10 questions cotées de 0 à 40 par ordre de gravité.

Une fibroscopie laryngée était réalisée par l'ORL pour objectiver la mobilité des cordes vocales, le degré d'occlusion et le défaut d'accolement des cordes vocales lors de la phonation (incompétence laryngée), et enfin l'existence éventuelle d'une asymétrie de la mobilité des plis vocaux.

Une réduction de la filière laryngée constituait un critère d'exclusion.

Les critères d'inclusion pour une proposition de médialisation par thyroplastie étaient les suivants : l'échec d'une rééducation orthophonique prolongée au delà de 10 mois, une fuite glottique supérieure à $2 \mathrm{cc} / \mathrm{s} / \mathrm{dB}$ conjuguée avec un temps maximum de phonation inférieur à 10 secondes, une dysphonie de grade 3, un Voice Handicap Index (VHI) égal ou supérieur à 20 , une réponse négative au test de masquage, et un défaut d'accolement laryngé en fibroscopie laryngée. 
Une femme de 62 ans (F) et un homme de 78 ans (H) présentant une maladie de Parkinson depuis plus de 8 ans avec une hypophonie et une dysarthrie ont été sélectionnés pour une médialisation laryngée.

Chez ces deux patients la présence d'une asymétrie de la mobilité des plis vocaux permettait de sélectionner le côté à médialiser.

La médialisation laryngée, effectuée sous anesthésie locale et neuroanalgésie, consistait à placer un implant de type Montgomery dans la corde vocale la plus déficitaire (5). Le placement optimal de l'implant et sa taille étaient déterminés en demandant au patient de produire des sons en peropératoire.

Les effets de la médialisation étaient objectivés en post opératoire (à J30 et J21 pour les deux patients, ainsi qu'à 5 mois pour le second patient) par les mêmes évaluations instrumentales et non instrumentales et par une fibroscopie laryngée (tableaux I, II).

On note un renforcement de l'adduction glottique se traduisant au niveau aérodynamique par une réduction de la fuite glottique dans des rapports allant d'un facteur 1.4 à 2 (figure 1) avec pour conséquence un allongement du temps maximum de phonation. Au niveau acoustique, on observe un doublement du nombre d'harmoniques avec un net enrichissement du timbre vocal.

Les évaluations non instrumentales montrent également une nette amélioration de la qualité ainsi que de l'efficacité vocale des patients évalués indirectement à travers le VHI 10 (figures 2 et 3). Lors de l'évaluation par le GRBAS, les 2 items nettement liés à l'hypophonie : B, le bruit de souffle et A, l'asthénie, présentent une amélioration importante (tableaux I, II). La respiration des patients n'apparait pas modifiée.

La fibroscopie postopératoire montre un meilleur accolement des deux plis vocaux en phonation pour les deux patients.

\section{Discussion}

Ainsi que l'ont décrit certains auteurs $(6,7,8,10)$, la médialisation améliore les qualités vocales des patients Parkinsoniens souffrant de dysphonie, par renforcement de l'adduction laryngée. Elle lève la barrière mécanique qui limite les effets de la prise en charge rééducative.

Une technique d'évaluation des paramètres acoustiques, aérodynamiques et laryngés couplée à des évaluations non instrumentales est apparue indispensable afin de définir avec rigueur les critères d'inclusion des patients. 
Les 2 patients présentés avaient suivi une rééducation orthophonique classique, d'une à deux séances hebdomadaires (pendant 12 mois et 36 mois), et nous étaient adressés en vue d'une prise en charge plus intensive de type LSVT L'expérience que nous avons acquise dans la pratique de cette méthode depuis son développement en France nous a conduits à en envisager les limites pour ces deux patients.

L'incompétence laryngée de ces deux patients se confirmant au cours des séances de type LSVT entreprises, nous avons abandonné la rééducation et proposé pour la première fois dans notre approche une médialisation.

Les analyses confirment les liens entre les paramètres aérodynamiques et acoustiques. Leur amélioration (tableaux, I, II) concorde avec ce qu'expriment les deux patients en termes d'amélioration de la qualité vocale et de la communication orale.

Les troubles articulatoires ne sont pas modifiés par ce traitement, mais la réduction de 1'hypophonie semble améliorer des aspects de l'intelligibilité, qui n'ont pas été évalués ici par un jury d'écoute, mais traduits très indirectement à travers les items du VHI 10. Afin d'apprécier les effets de la réduction de l'hypophonie sur l'intelligibilité de la parole, un test spécifique d'évaluation de ce paramètre devra être pratiqué lors des prises en charge ultérieures. De même l'élargissement de l'échantillon de patients traités permettra d'appliquer les tests de corrélation nécessaires à une évaluation pertinente de cette technique.

Comparativement aux techniques de médialisation par injection d'une substance hétérologue non réversible décrites auprès des patients parkinsoniens $(6,7,8,10)$ ou même de graisse autologue dans d'autres types de pathologie (5), la thyroplastie semble réunir, pour cette population, différents avantages : la réalisation sous anesthésie locale, l'ajustement de la taille de l'implant en fonction du résultat fonctionnel en peropératoire et surtout par rapport aux injections cordales, la possibilité de retirer l'implant dans le cas où des troubles de la mobilité des plis vocaux entraineraient une dyspnée laryngée. Face à l'absence de littérature concernant les modifications laryngées après extrusion de l'implant de Montgomery, la réversibilité de la médialisation par thyroplastie demeure un atout majeur dans ce contexte de pathologie évolutive.

\section{Conclusion}

La médialisation laryngée permet d'améliorer l'adduction laryngée et renforce l'intensité vocale chez des patients atteints de maladie de Parkinson. Si elle ne joue pas sur la dysarthrie elle-même, la réduction de l'hypophonie, analysée à partir d'outils d'évaluations objective et 
subjective, améliore la qualité vocale et d'une manière plus générale, la communication orale et la vie sociale des patients.

La thyroplastie, faite sous anesthésie locale, avec un ajustement possible peropératoire, représente une technique de médialisation adaptée au caractère potentiellement évolutif de la maladie de Parkinson, l'implant pouvant en effet être retiré.

Déclaration d'intérêts : les auteurs déclarent ne pas avoir de conflits d'intérêts en relation avec cet article 


\section{Références}

1 - Darley FL, Aronson AE, Brown JR. Motor Speech Disorders, Philadelphia:WB Sanders Co. 1975.

2 - Rusz J, Cmejla R, Ruzickova H, Ruzicka E. Quantitative acoustic measurements for characterization of speech and voice discorders in early untreated Parkinson's disease. J Acoust Soc Am. 2011;129:350-367.

3 - Sapir S, Spielman JL, Ramig LO, Story BH, Fox C. Effects of intensive voice treatment (the LSVT) on vowel articulation in dysarthric individuals with idiopathic Parkinson disease : acoustic and perceptual findings. J Speech Lang Hear Res. 2007;50:899-912.

4 - Ramig LO, Dromey C. Aerodynamic mechanisms underlying treatment-related changes in vocal intensity in patients with Parkinson disease. J Speech Hearing Res. 1996 39:798-807.

5 - Cannito MP, Suiter DM, Beverly D, Chorma L, Wolf T, Pfeiffer RM. Sentence intelligibility before and after voice treatment in speakers with idiopathic Parkinson's disease. J Voice. 2012;26:214-219.

6 - Berke GS, Gerratt B, Kreiman J, Jackson K. Treatment of Parkinson hypophonia with percutaneous collagen augmentation. Laryngoscope. 1999;109: 1295-1299.

7 - Kim SH, Kearney JJ, Atkins JP. Percutaneous laryngeal augmentation for treatment of parkinsonian hypophonia. Otolaryngol Head Neck Surg. 2002 ;126: 653-656.

8 - Hill AN, Jankovic J, Vuong KD, Donova,n D. Treatment of hypophonia with collagen vocal cord augmentation in patients with parkinsonian. Mov Disod. 2003;18 : 1190-1192. 9 - Sewall GK, Jiang J and Ford CN. Clinical evaluation of Parkinson's-related dysphonia. Laryngooscope. 2006;116: 1740-1744.

10 - Remacle M and Lawson G. Results with collagen injection into the vocal folds for medicalization. Curr Opin Otolaryngol Head Neck Surg. 2007;15: 148-152.

11 - Périé S, Roubeau B, Liesenfelt I, Debono G, Bruel M, Lacau St Guily J. Role of medialization in the improvement of breath in unilatral vocal fold paralysis. Ann Otol Rhinol Larungol. $2002 ; 111: 1026-1033$. 
12 - Dejonckere P, Obbens C, De Moor G M, Wieneke, (1993). Perceptual evaluation of dysphonia: Reliability and relevance.Folia Phoniatrica. 1993;45: 76-83.

13 - Kreiman J, Gerrat BR, Kempster G B, Erman A, and Berke GS. Perceptual Evaluation of Voice Quality : Review, Tutorial, and a Framework for Future Research, Journal of Speech and Hearing Research. 1996;36:21-40.

14 - Rosen CA, Lee AS, Osborne J, Zullo T, Murry T. Development and validation of the voice handicap index-10. Laryngoscope. 2004;114:1549-1556. 
Figure 1 : Evaluation pré et post opératoire de la fuite glottique. Sujets féminin (F) et masculin (M)

Figure 2 : Evaluation pré et post opératoire du GRBAS cumulé. Sujets féminin (F) et masculin (M)

Figure 3 : Evaluation pré et post opératoire du VHI 10 (Voice Handicap Index). Sujets féminin $(\mathrm{F})$ et masculin $(\mathrm{M})$ 
Tableau I. Evaluation vocale pré et post opératoire chez le sujet féminin

\begin{tabular}{lcc}
\hline femme & Pré Op & Post Op (J30) \\
\hline Nb d'harmoniques & 5 & 6 à 12 \\
Fuite glottique & $2.57 \mathrm{cc} / \mathrm{s} / \mathrm{dB}$ & $1.8 \mathrm{cc} / \mathrm{s} / \mathrm{dB}$ \\
TMP (s) et volume & $7.2 \mathrm{~s} \mathrm{et} 1.4 \mathrm{dm} 3$ & $12.3 \mathrm{~s} \mathrm{et} 1.5 \mathrm{dm} 3$ \\
Phrase (volume) & $0.45 \mathrm{dm} 3$ & $0.52 \mathrm{dm} 3$ \\
\hline GRBAS & 30330 & 11110 \\
VHI 10 & $30 / 40$ & $14 / 40$ \\
\hline
\end{tabular}

Tableau II. Evaluation vocale pré et post opératoire chez le sujet masculin

\begin{tabular}{lccc}
\hline homme & Pré Op & Post Op (J21) & Post Op (5 mois) \\
\hline Nb d'harmoniques & 6 & 18 & 10 \\
Fuite glottique & $3.55 \mathrm{cc} / \mathrm{s} / \mathrm{dB}$ & $1.7 \mathrm{cc} / \mathrm{s} / \mathrm{dB}$ & $1,66 \mathrm{cc} / \mathrm{s} / \mathrm{dB}$ \\
TMP (s) et volume & $7.6 \mathrm{~s} \mathrm{et} 2 \mathrm{dm} 3$ & $26 \mathrm{~s} \mathrm{et} 2 \mathrm{dm} 3$ & $22,4 \mathrm{~s} \mathrm{et} 3 \mathrm{dm} 3$ \\
Phrase (volume) & $0.7 \mathrm{dm} 3$ & $0.3 \mathrm{dm} 3$ & $0,6 \mathrm{dm} 3$ \\
\hline GRBAS & 31231 & 11111 & 22111 \\
VHI 10 & $20 / 40$ & - & $7 / 40$ \\
\hline
\end{tabular}


Figure 1 : Evaluation pré et post opératoire de la fuite glottique. Sujets féminin (F) et masculin (M)

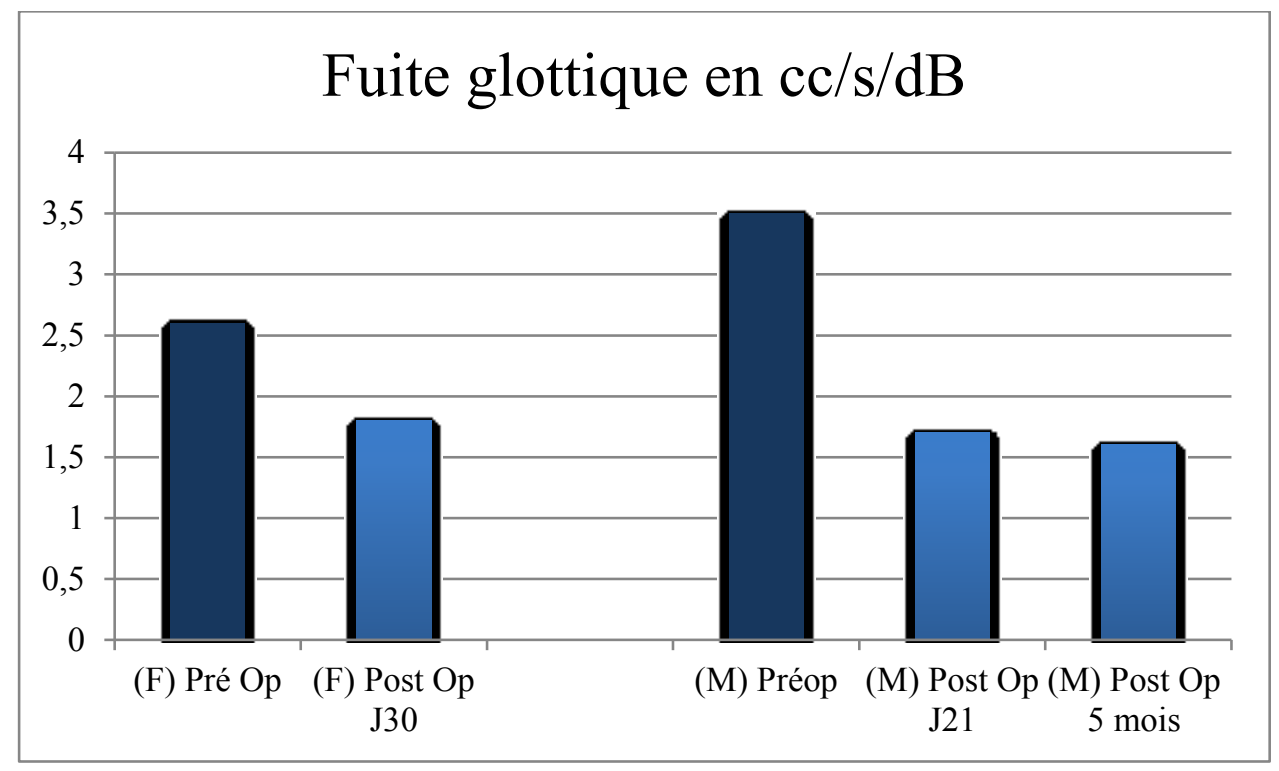


Figure 2 : Evaluation pré et post opératoire des GRBAS cumulés. Sujets féminin (F) et masculin (M)

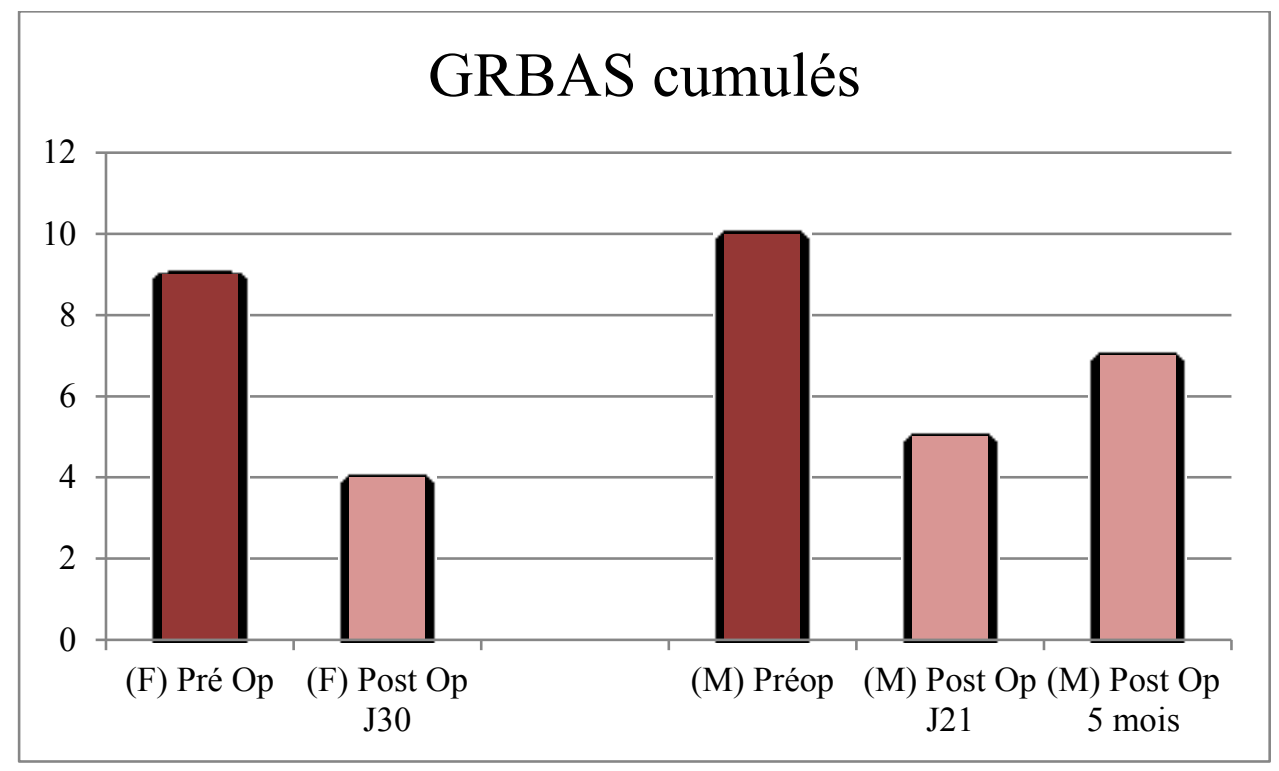


Figure 3 : Evaluation pré et post opératoire du VHI 10 (Voice Handicap Index). Sujets féminin (F) et masculin (M)

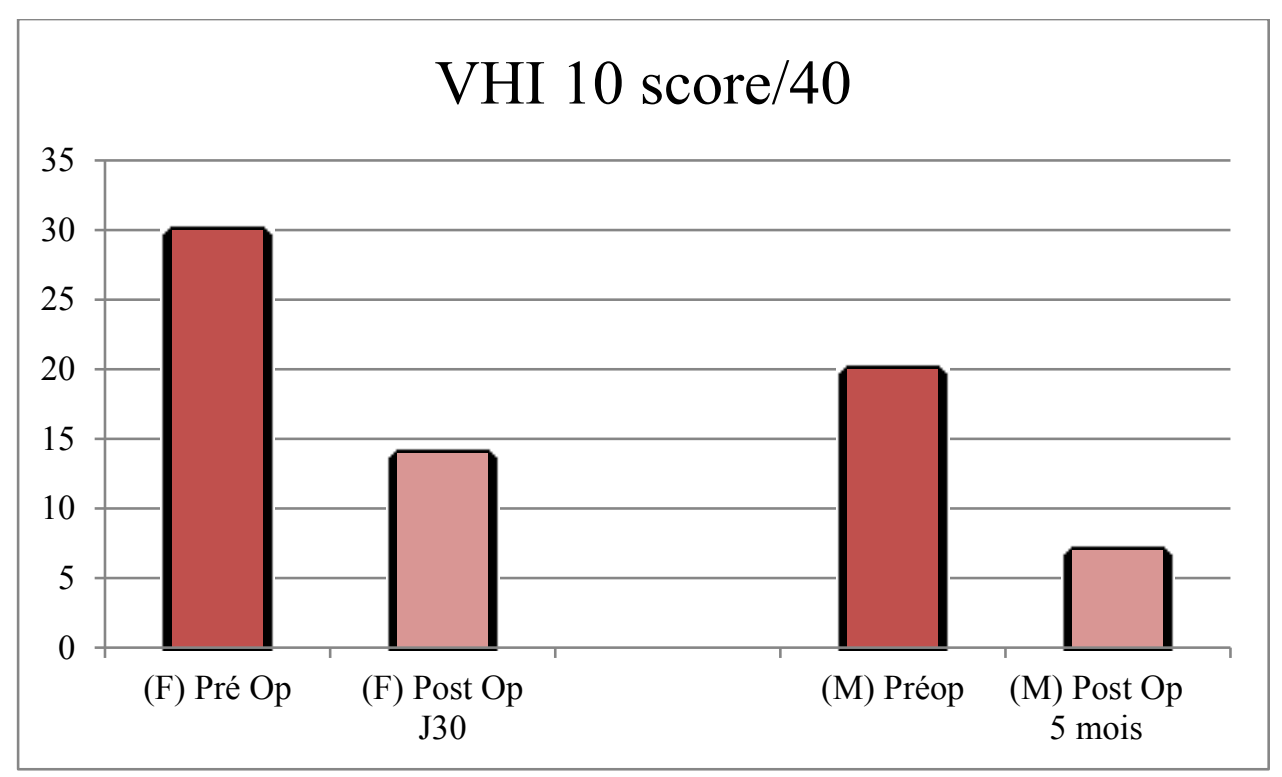

